\title{
Signos Neurológicos Blandos y procesos cognitivos en niños escolares mexicanos de 6-11 años
}

\author{
Judith Salvador-Cruz; Dulce Saraí Tovar Vital; Abraham Segura Villa; Luis Ledesma-Amaya; Antonio García Anacleto; \\ Cristina Aguillón Solis; Eric Sánchez Vielma; Marlene Rodríguez Martínez
}

How to cite this article:

Salvador-Cruz, J., Tovar, D.S., Segura, A., Ledesma, L., García, A., Aguillón, C., Sánchez, E., \& Rodríguez, M. (2019). Neurological Soft Signs and cognitive processes in Mexican schoolchildren aged 6 to 11 years. Acta Colombiana de Psicología, 22(2), 28-40. doi: http://www.doi.org/10.14718/ACP.2019.22.2.3

Recibido, septiembre 17/2017; Concepto de evaluación, noviembre 10/2017; Aceptado, octubre 17/2018

\author{
Judith Salvador-Cruz \\ Universidad Nacional Autónoma de México, México \\ ORCID: https://orcid.org/0000-0003-1902-1525 \\ Dulce Saraí Tovar Vital \\ Universidad Nacional Autónoma de México, México \\ ORCID: https://orcid.org/0000-0002-7722-0103 \\ Abraham Segura Villa \\ Universidad Nacional Autónoma de México, México \\ ORCID: https://orcid.org/0000-0002-1008-985X \\ Luis Ledesma-Amaya** \\ Universidad Autónoma de Baja California, México \\ ORCID: https://orcid.org/0000-0002-2780-272X \\ Antonio García Anacleto \\ Universidad Nacional Autónoma de México, México \\ ORCID: https://orcid.org/0000-0001-6005-9379 \\ Cristina Aguillón Solis \\ Universidad Nacional Autónoma de México, México \\ ORCID: https://orcid.org/0000-0002-0785-0249 \\ Eric Sánchez Vielma \\ Universidad Nacional Autónoma de México, México \\ ORCID: https://orcid.org/0000-0002-9062-6511 \\ Marlene Rodríguez Martínez \\ Universidad Nacional Autónoma de México, México \\ ORCID: https://orcid.org/0000-0002-9912-8500
}

\begin{abstract}
Resumen
Los Signos Neurológicos Blandos (SNB) son indicativos de interrupciones generalizadas en las redes de trabajo neuronal de áreas cortico-subcorticales, cuya presencia conlleva a problemas en el desarrollo neurocognitivo del niño que representan repercusiones académicas negativas. En la presente investigación se evaluó y comparó la presencia de SNB con los procesos cognitivos de 144 participantes mexicanos de estrato socioeconómico medio-bajo con edades entre los 6 y 11 años sin antecedentes neurológicos o psiquiátricos por medio de los Cuestionarios de Madurez Neuropsicológica (CUMANIN) y Madurez Neuropsicológica Escolar (CUMANES). Los resultados indicaron diferencias significativas por sexo, ya que las niñas presentaron mejor desempeño en la articulación de palabras. En los grupos por edad, las diferencias significativas se encontraron en leximetría-comprensión, visopercepción y función ejecutiva-errores; y, en general, los participantes mostraron presencia de SNB de desarrollo, que incluyen afectaciones en: lenguaje (problemas articulatorios, alteraciones del lenguaje oral y escrito, dificultad para encontrar palabras), psicomotricidad, visopercepción y otras funciones cognitivas. Al final, se concluye que la identificación y diagnóstico temprano de los SNB permite disminuir el riesgo de fracaso escolar. Palabras clave: signos neurológicos blandos, integración sensorial, desarrollo neurocognitivo, escolares, infancia.
\end{abstract}

* Facultad de Estudios Superiores Zaragoza, UNAM, Laboratorio de Neuropsicología del Desarrollo: alteraciones neurológicas, psiquiátricas y rehabilitación. Dir.: C/ Batalla 5 de mayo s/n. Esquina Fuerte de Loreto. Colonia Ejército de Oriente, 09230, México D. F., México. Tel.: (55) 577363 30. salvadcj@gmail.com

** Escuela de Ingeniería y Negocios, Guadalupe Victoria. Laboratorio de Neurociencias y Cognición. Colaborador del Cuerpo Académico Salud Mental Profesión y Sociedad.

Agradecemos al proyecto PAPIIT IN 306116 por el financiamiento otorgado para realizar esta investigación. 


\title{
Neurological Soft Signs and cognitive processes in Mexican schoolchildren aged 6 to 11 years
}

\begin{abstract}
Abstrac
Neurological Soft Signs (NSS) are indicative of generalized disruptions in neurological networks of cortico-subcortical areas. Their presence leads to problems in children's cognitive development with future academic repercussions. The presence of NSS was assessed and compared in 144 children aged 6 to 11 years of low-medium socioeconomic status from Mexico City and the metropolitan area through the Infant Neuropsychological Maturity Questionnaire (CUMANIN, for its Spanish acronym) and the School Neuropsychological Maturity Questionnaire (CUMANES, for its Spanish acronym). Results indicate significant differences by sex. Girls showed better performance in word articulation. In groups by age, significant differences were found in leximetriccomprehension, visual perception and executive function-errors. Participants showed the presence of developmental NSS which include: language (articulation disorders, oral and written language disturbances, difficulty finding words), psychomotricity, visual perception and other cognitive functions. There are several factors related to those impairments such as age, socioeconomic context and critical stages in child's development. Identification and early diagnosis can reduce the risk of school failure.

Key words: Neurological soft signs, sensory integration, neurocognitive development, schoolchildren, childhood.
\end{abstract}

\section{Sinais Neurológicos Sutis e processos cognitivos em crianças escolares mexicanas de 6 a 11 anos}

\author{
Resumo
}

\begin{abstract}
Os Sinais Neurológico Sutis (SNS) são indicadores de interrupções generalizadas nas redes de trabalho neural de áreas córticosubcorticais, cuja presença leva a problemas no desenvolvimento neurocognitivo da criança que representam repercussões acadêmicas negativas. Na presente pesquisa, foi avaliada e comparada a presença de SNS com os processos cognitivos de 144 participantes mexicanos de estrato socioeconômico médio-baixo com idades entre 6 e 11 anos, sem antecedentes neurológicos ou psiquiátricos, por meio dos Cuestionarios de Madurez Neuropsicológica (CUMANIN) e Madurez Neuropsicológica Escolar (CUMANES). Os resultados indicaram diferenças significativas por gênero, já que as meninas apresentaram melhor desempenho na articulação de palavras. Nos grupos por idade, as diferenças significativas foram encontradas na velocidade de leitura e compreensão, visuopercepção e função executiva e erros. Em geral, os participantes mostraram presença de SNS de desenvolvimento, que incluem interferências na linguagem (problemas articulatórios, alterações da linguagem oral e escrita, dificuldade para encontrar palavras), psicomotricidade, visuopercepção e outras funções cognitivas. Ao final, conclui-se que a identificação e o diagnóstico precoce dos SNS permite diminuir o risco de fracasso escolar.

Palavras-chave: sinais neurológicos sutis, integração sensorial, desenvolvimento neurocognitivo, escolares, infância.
\end{abstract}

\section{Introducción}

Los Signos Neurológicos Blandos (SNB) no son localizables de forma exacta en el sistema nervioso (Chan et al., 2016; Bombin, Arango \& Buchanan, 2005), sino que (a) se detectan por medio de un examen neurológico clínico en ausencia de características de un trastorno fijo o transitorio (lesión neurológica); (b) se asumen como un déficit no localizable en algún área del sistema nervioso central (Malhotra, Borade, Sharma, Satija \& Gunjan, 2017); y (c) se evidencian mediante alteraciones en funciones motoras, sensitivas y de integración (Bombin et al., 2005).

Específicamente, los SNB comprenden un amplio rango de sutiles déficits neurológicos relacionados con la integración perceptivo-sensorial, la coordinación motriz, el balance, la secuenciación de actos motores complejos, el exceso de movimientos y, ocasionalmente, la torpeza y la ocurrencia de reflejos primitivos (Hirjak et al., 2017).
Como tal, los SNB se consideran signos iniciales importantes de un trastorno evolutivo con origen en el desarrollo neurológico (Ojagbemi, 2017), por lo que resulta importante realizar la evaluación y detección de los SNB en etapas tempranas del desarrollo, ya que es en estas cuando hay mayor riesgo de presentarse. Sin embargo, es un hecho que estas dificultades en el neurodesarrollo pasan inadvertidas por gran parte de la población, y esto termina siendo un factor predictor de fracaso y deserción escolar (Mateos, 2009).

Para autores como Faruk-Demirel, Demirel, TayibbKadak, Emül y Duran (2016), los SNB son un factor de vulnerabilidad inespecífico asociado a distintos trastornos psicológicos y psiquiátricos, entre los que se encuentran, entre otros, la esquizofrenia (Chan et al., 2016; Chrobak et al., 2016; Emsley et al., 2017; Mithun, Kamal, Aparajeeta \& Subrata, 2016; Papiol, Fatjo-Vilas \& Shulze, 2016), el trastorno obsesivo compulsivo (Chetail-Vijay \& Shubhangi, 2016; Dhuri \& Parkar, 2016; Peng et al., 2012) y el trastorno por déficit de atención e hiperactividad (Abdel-Aziz, 
El Sheikh, Mohsen, Khalil \& Hassan, 2016; Cardo, Casanovas, Banda \& Servera, 2008; Jingbo-Gong, JingtaoXie, Yajie-Zhang \& Su-Hong-Wang, 2015); trastornos que conllevan considerables resultados negativos en el aspecto psicosocial y que, incluso, afectan procesos mentales como el de la cognición social (Pitizianti et al., 2017).

En estudios recientes se ha encontrado evidencia sobre que la manifestación de SNB tiene una relación estrecha con la presencia de dificultades en el aprendizaje-como en la lectura (Poblano, Borja, Elías, García-Pedroza \& Arias, 2002) - y con un bajo coeficiente intelectual durante la niñez y la adolescencia (Manaut-Gil, Vaquero-Casares, QuinteroGallego, Pérez-Santamaría \& Gómez-González, 2004). Por ejemplo, en un estudio con pacientes con discapacidad específica del aprendizaje (DEA) se observó que el 58.6\% presentaba SNB $(n=100)$, acompañados de anomalías en la coordinación motriz (disdiadocosinesia) y la extinción sensorial (Somale, Kondekar, Rathi \& Iyer, 2016). Y, en otro trabajo, con pacientes con TDAH (Patankar, Sangle, Henal, Shah \& Kamath, 2012), se observó que el $84 \%$ de ellos presentaba SNB $(n=52)$, de los cuales el $34.6 \%$ contaba con diagnóstico de DEA, como el de dislexia.

Por otra parte, dentro de los factores atribuidos al bajo rendimiento escolar se encuentra que en al menos uno de cada cinco casos subyace un componente neurodisfuncional derivado de alteraciones congénitas, noxas perinatales, retardo neuromadurativo o disfunción del sistema nervioso (TorresGonzález, Salvador-Cruz, Flores \& Ricardo-Garcell, 2016).

Por otra parte, se ha encontrado que en la presencia de SNB en niños con nivel socioeconómico bajo, pueden repercutir condiciones de poca estimulación ambiental y un mayor riesgo de complicaciones pre y postnatales, así como una mala nutrición y enfermedades durante el crecimiento (TorresGonzález, Salvador-Cruz, Flores \& Ricardo-Garcell, 2016).

Al respecto, en México, por ejemplo, la población entre 5 y 14 años de edad es de aproximadamente 20 millones (INEGI, 2015), y de esta se estima que cerca del $15 \%$ presenta problemas en el desarrollo del sistema nervioso; hecho que tiene considerables repercusiones en el ámbito escolar y afectivo de los niños (Lipina, 2016; Salvador-Cruz et al., 2016; Torres-González et al., 2016), y que, junto con los antecedentes presentados, justifica la relevancia del estudio de los SNB en diversos contextos, sobre todo en aquellos en los que la población infantil presenta factores sociodemográficos privativos y se encuentra más vulnerable, es decir, que pertenezcan a un estrato socioeconómico bajo.

En relación con lo anterior, estudios realizados desde mediados del siglo XX han demostrado que la condición de pobreza compromete en forma significativa el desarrollo cognitivo y emocional infantil (Hermida, Segretin, Lipina, Benarós \& Colombo, 2010), y es por esto que en la literatura han predominado abordajes que se apoyan de paradigmas psicométricos y educativos. Sin embargo, desde el enfoque de las neurociencias, algunas investigaciones demuestran el papel que ejerce la condición socioeconómica en el desempeño de tareas cognitivas en áreas atencionales, de lenguaje, funcionamiento ejecutivo y procesos mnésicos (Hermida et al., 2010; Mazzoni, Stelzer, Cervigni \& Martino, 2014).

Finalmente, cabe mencionar que, de acuerdo con la UNICEF (s. f.), en el 2014 una de cada dos niñas y adolescentes en México padecía pobreza y una de cada nueve se encontraba en pobreza extrema; además de que el $53.9 \%$ de la población de 0 a 17 años en México (21.4 millones) carecía de las condiciones mínimas de educación, acceso a la salud, seguridad social o vivienda de calidad -con carencias de servicios básicos y de alimentación-.

Teniendo todo lo anterior en cuenta, y dado el impacto social que esto conlleva y sus repercusiones en el desarrollo normal del sistema nervioso, el objetivo del presente estudio fue evaluar y comparar la presencia de SNB con los procesos cognitivos superiores de niños escolares mexicanos de un estrato socioeconómico medio-bajo sin presencia de antecedentes neurológicos o psiquiátricos.

\section{Método}

Para el presente estudio se utilizó un diseño no experimental, transversal y descriptivo (Kerlinger, 2002).

\section{Participantes}

Se seleccionó una muestra de manera no probabilística, intencional y por cuotas (Kerlinger, 2002), conformada por 144 niños mexicanos de estrato socioeconómico mediobajo, 75 hombres y 69 mujeres, con edades de 6 a 11 años (en la Tabla 1 se puede observar la distribución por edad y sexo). Se seleccionaron participantes de seis escuelas primarias públicas de la Ciudad de México y del área conurbada, teniendo en cuenta los criterios de exclusión: (a) que mencionaran antecedentes metabólicos, neurológicos o

Tabla 1.

Distribución de la muestra según sexo y edad

\begin{tabular}{ccccccc}
\hline \multirow{2}{*}{$\begin{array}{c}\text { Edad } \\
\text { (años) }\end{array}$} & \multicolumn{2}{c}{ Niños } & \multicolumn{2}{c}{ Niñas } & \multicolumn{2}{c}{ Total } \\
\cline { 2 - 7 } & $\mathrm{N}$ & $\%$ & $\mathrm{~N}$ & $\%$ & $\mathrm{~N}$ & $\%$ \\
\hline 6 & 19 & 13.19 & 23 & 15.97 & 42 & 29.17 \\
7 & 13 & 9.03 & 13 & 9.03 & 26 & 18.06 \\
8 & 18 & 12.50 & 17 & 11.81 & 35 & 24.31 \\
9 & 6 & 4.17 & 8 & 5.56 & 14 & 9.72 \\
10 & 10 & 6.94 & 5 & 3.47 & 15 & 10.42 \\
11 & 9 & 6.25 & 3 & 2.08 & 12 & 8.33 \\
\hline Total & 75 & 52.08 & 69 & 47.92 & 144 & 100 \\
\hline
\end{tabular}


psiquiátricos, alteraciones auditivas o visuales no corregidas; y (b) que no concluyeran el cuestionario de antecedentes neurológicos y psiquiátricos (Salvador \& Galindo, 1996).

\section{Instrumentos}

Se utilizó el Cuestionario de Madurez Neuropsicológica Infantil (CUMANIN), de Portellano, Mateos y Martínez (2009), el Cuestionario de Madurez Neuropsicológica Escolar (CUMANES), de Portellano, Mateos y Martínez (2012), y un cuestionario adicional de antecedentes neurológicos y psiquiátricos.

Cuestionario de Madurez Neuropsicológica Infantil (CUMANIN). Este cuestionario, desarrollado por Portellano et al. (2009), es un instrumento de madurez neuropsicológica para la edad preescolar diseñado para niños de entre 3 y 6 años, conformado por ocho escalas principales (Psicomotricidad, Lenguaje articulatorio, Lenguaje comprensivo, Lenguaje expresivo, Estructuración espacial, Visopercepción, Memoria icónica y Ritmo) y cinco escalas auxiliares (Atención, Fluidez verbal, Lectura, Escritura y Lateralidad); y validado en población española con una consistencia interna entre $r=.71$ y .92 en sus diferentes subescalas. Para la estimación de confiabilidad del instrumento, en el presente estudio se obtuvo la medida de consistencia interna, en donde se encontró un alfa de Cronbach de $r=.690$.

Cuestionario de Madurez Neuropsicológica Escolar (CUMANES). Esta prueba, desarrollada por Portellano et al. (2012), permite valorar de un modo extenso el desarrollo cognitivo de niños entre los 7 y los 11 años en seis dominios o áreas diferentes: Lenguaje, Visopercepción, Función ejecutiva, Memoria, Ritmo y Lateralidad. Con respecto a su fiabilidad, el instrumento obtuvo en población española un alfa de Cronbach de $r=.79$, y su consistencia interna osciló entre $r=.61$ y .85 en las diferentes subescalas que lo conforman. En el presente estudio se evaluó la consistencia interna en la muestra, donde se obtuvo un alfa de Cronbach de $r=.736$.

Ambas evaluaciones permiten determinar el grado de madurez neuropsicológica, así como la posible presencia de signos de disfunción cerebral.

Cuestionario de antecedentes neurológicos y psiquiátricos. Adicionalmente, se utilizó un cuestionario breve compuesto por diez preguntas con respuestas dicotómicas con el fin de recopilar los datos neurológicos, psiquiátricos, prenatales, perinatales, postnatales y de dificultades de aprendizaje de los participantes (Salvador \& Galindo, 1996).
Procedimiento

El proceso de evaluación se realizó bajo la autorización de los responsables de las instituciones educativas visitadas. Teniendo esta autorización, se le entregó a los padres de familia voluntarios un cuestionario de antecedentes neurológicos y psiquiátricos (Salvador \& Galindo, 1996) con el objetivo de identificar a los niños que cumplían con los criterios requeridos para la presente investigación; y una vez identificados, se procedió a realizar la evaluación correspondiente, de aproximadamente 40 minutos, llevada a cabo dentro del contexto escolar.

El procedimiento de evaluación para los grupos de edad se dividió en dos: (a) niños de 6 años, a quienes se les aplicó el Cuestionario de Madurez Neuropsicológica Infantil (CUMANIN; Portellano et al., 2009); y (b) niños de 7 a 11 años, que contestaron el Cuestionario de Madurez Neuropsicológica Escolar (CUMANES; Portellano et al., 2012).

Dentro de las consideraciones éticas se tuvo en cuenta que todos los padres o tutores de los niños firmara el consentimiento informado por escrito, y que todos los participantes dieran cuenta de su aquiescencia de manera verbal con el hecho de hacer parte del estudio.

\section{Análisis de datos}

Las puntuaciones centiles de las subpruebas del CUMANIN y del CUMANES informan la posición de las puntuaciones directas obtenidas con respecto a todo el conjunto de observaciones realizadas durante la validación de la prueba; así, las puntuaciones centiles se toman como una medida que identifica dificultades durante la ejecución de cada una de las subpruebas. Acá, a mayor posición centil, menores son las dificultades, y viceversa.

Para estimar la centralidad y dispersión de los puntajes de las subescalas que evalúan los SNB se realizó una descripción de las subescalas aplicadas por medio de medianas, medias y desviaciones estándar; mientras que para las comparaciones por sexo y edad se tuvieron en cuenta las clasificaciones por centiles (CUMANIN) y decatipos (CUMANES) según la ejecución de los participantes. Finalmente, se utilizaron pruebas estadísticas no paramétricas debido a la falta de normalidad en las distribuciones de cada variable medida en los instrumentos utilizados, y debido a que fueron evaluadas por medio de la prueba de normalidad de Kolmogorov-Smirnov (K-S); por tanto, se utilizaron las pruebas U de Mann-Whitney y Kruskall-Wallis para comparar las diferencias con respecto a las variables de sexo y edad (Coolican, 2014). Todos los análisis se llevaron a cabo por medio del programa estadístico informático SPSS ${ }^{\circledR}$ v. 20.

El análisis cualitativo utilizado para caracterizar las problemáticas reportadas se realizó con los lineamientos propuestos por los autores de los instrumentos de evaluación CUMANIN 
(Portellano etal., 2009) y CUMANES (Portellano et al., 2012), presentados en el manual de cada instrumento de evaluación.

\section{Resultados}

A continuación se presentan los resultados descriptivos divididos por las escalas principales de los instrumentos de medición de madurez neuropsicológica infantil (CUMANIN) y escolar (CUMANES).

En la Tabla 2 se puede observar los SNB encontrados en los niños de 6 a 11 años, donde resaltan la alteración en la integración sensoperceptual, la coordinación motriz y la orientación izquierda-derecha.

Tabla 2.

Signos Neurológicos Blandos presentes en los niños de 6 a 11 años

\begin{tabular}{cl}
\hline Clasificación & \multicolumn{1}{c}{ Signo neurológico } \\
\hline \multirow{3}{*}{ Motores } & \multicolumn{1}{c}{ Integración sensorial } \\
& $\begin{array}{l}\text { Disdiadococinesia } \\
\text { Trastorno de coordinación } \\
\text { Trastorno de motricidad fina y gruesa } \\
\text { Impersistencia motora }\end{array}$ \\
& Prensión inmadura del lápiz \\
& Trastorno de orientación espacial \\
Perceptivos & $\begin{array}{l}\text { Dificultad para el reconocimiento de } \\
\text { derecha-izquierda }\end{array}$ \\
& Dificultades de aprendizaje \\
& Dificultades de atención sostenida \\
& Alteración en procesos cognitivos: memoria y \\
& razonamiento \\
& Problemas del lenguaje oral o escrito \\
& Problemas en lenguaje articulado \\
\hline
\end{tabular}

CUMANIN (6 años)

Se realizó un análisis de medidas de tendencia central y de dispersión de los centiles de cada subprueba del CUMANIN para el primer grupo de edad con el objetivo de identificar las subpruebas con mayor y menor puntuación centil media. A partir de esto, se observó que la subprueba estructuración espacial obtuvo la mayor puntuación centil media $(M=65.88)$, mientras que psicomotricidad obtuvo la puntuación menor $(M=31.48)$. Los resultados del CUMANIN se pueden observar en la Tabla 3.

Tabla 3.

Resultados de los centiles obtenidos por los niños de 6 años en el CUMANIN

\begin{tabular}{lccl}
\hline \multicolumn{1}{c}{ Subprueba } & $\mathrm{M}(\mathrm{DE})$ & $\mathrm{M}_{\mathrm{e}}$ & \multicolumn{1}{c}{ K-S } \\
\hline Psicomotricidad & $31.48(30.49)$ & 20 & $1.876^{*}$ \\
Lenguaje articulatorio & $42.02(28.54)$ & 40 & 1.301
\end{tabular}

\begin{tabular}{llll} 
Lenguaje expresivo & $35.60(27.59)$ & 40 & $1.595^{*}$ \\
Lenguaje comprensivo & $45.05(31.30)$ & 35 & 1.333 \\
Estructuración espacial & $65.88(29.98)$ & 88 & $1.759^{*}$ \\
Visopercepción & $37.07(34.54)$ & 22.5 & $1.546^{*}$ \\
Memoria icónica & $65.21(28.70)$ & 70 & 1.275 \\
Ritmo & $36.64(29.87)$ & 35 & 1.205 \\
Fluidez verbal & $39.29(27.30)$ & 37.5 & 0.949 \\
Atención & $39.50(27.05)$ & 45 & $1.25^{*}$ \\
Lectura & $55.24(24.24)$ & 50 & $0.865^{*}$ \\
Escritura & $50.33(25.07)$ & 45 & $0.798^{*}$ \\
\hline
\end{tabular}

Nota $. \mathrm{M}_{\mathrm{e}}=$ Mediana; $\mathrm{M}=$ media; $\mathrm{DE}=$ desviación estándar; $\mathrm{K}-\mathrm{S}=$ $\mathrm{z}$ de Kolmogorov-Smirnov. * significancia $<.05$ (no homogénea).

En la Tabla 4 se presenta el porcentaje del rendimiento de los niños según su ejecución en cada una de las subpruebas de CUMANIN. Dichas clasificaciones se dividieron en rendimiento bajo para puntajes por debajo del percentil 40 , rendimiento medio para puntajes entre el percentil 40 y 60 , y rendimiento alto para puntajes del percentil 60 al 100 (Portellano et al., 2009). Con esta clasificación, se observó que el porcentaje de participantes que obtuvieron un rendimiento bajo fue de $64.3 \%$ en psicomotricidad, $64.3 \%$ en ritmo, $57.1 \%$ en visopercepción; 52.4 en lenguaje comprensivo y $50.0 \%$ en fluidez verbal.

Tabla 4.

Porcentaje de participantes con rendimiento bajo, medio y alto en las subpruebas del CUMANIN (6 años)

\begin{tabular}{llll}
\hline \multirow{2}{*}{\multicolumn{1}{c}{ Subprueba }} & \multicolumn{3}{c}{ Rendimiento } \\
\cline { 2 - 4 } & Bajo (\%) & Medio (\%) & Alto (\%) \\
\hline Psicomotricidad & 64.3 & 9.5 & 26.2 \\
Lenguaje articulatorio & 47.6 & 33.3 & 19.0 \\
Lenguaje expresivo & 47.6 & 33.3 & 19.0 \\
Lenguaje comprensivo & 52.4 & 11.9 & 35.7 \\
Estructuración espacial & 23.8 & 23.8 & 52.4 \\
Visopercepción & 57.1 & 9.5 & 33.3 \\
Memoria icónica & 14.3 & 35.7 & 50.0 \\
Ritmo & 64.3 & 0.0 & 35.7 \\
Fluidez verbal & 50.0 & 31.0 & 19.0 \\
Atención & 47.6 & 35.7 & 16.7 \\
Lectura & 23.8 & 35.7 & 40.5 \\
Escritura & 33.3 & 28.6 & 38.1 \\
\hline
\end{tabular}

Por otra parte, se utilizó la prueba U de Mann-Whitney para identificar la existencia de diferencias entre las ejecucionesen las puntuaciones centiles según la variable sexo, donde se encontraron diferencias significativas solo en la subprueba de lenguaje expresivo, ya que las niñas mostraron un mejor desempeño.

Con respecto a la descripción cualitativa de la ejecución por parte del grupo de participantes que obtuvieron puntuaciones centiles bajas en las subescalas del CUMANIN, es importante señalar que los problemas principales se encontraron en: 
Salvador-Cruz, J., Tovar Vital, D.S., Segura Villa, A., Ledesma-Amaya, L., García Anacleto, A., Aguillón Solis, C.,

Tabla 5.

Rangos de ejecución en el CUMANIN según el sexo (6 años)

\begin{tabular}{|c|c|c|c|c|}
\hline \multirow{3}{*}{ Subprueba } & \multicolumn{2}{|c|}{ Sexo } & \multirow{3}{*}{ U de Mann-Whitney } & \multirow{3}{*}{$\mathrm{p}$} \\
\hline & \multicolumn{2}{|c|}{ (rango promedio) } & & \\
\hline & Niños $^{\mathrm{a}}$ & Niñas $^{\mathrm{b}}$ & & \\
\hline Psicomotricidad & 20.87 & 22.02 & 206.5 & .758 \\
\hline Lenguaje articulatorio & 18.97 & 23.59 & 170.5 & .217 \\
\hline Lenguaje expresivo & 17.29 & 24.98 & 138.5 & $.037 *$ \\
\hline Lenguaje comprensivo & 19.08 & 23.5 & 172.5 & .24 \\
\hline Estructuración espacial & 23.42 & 19.91 & 182 & .349 \\
\hline Visopercepción & 19.66 & 23.02 & 183.5 & .375 \\
\hline Memoria icónica & 18.82 & 23.72 & 167.5 & .191 \\
\hline Ritmo & 22.24 & 20.89 & 204.5 & .718 \\
\hline Fluidez verbal & 22.66 & 20.54 & 196.5 & .575 \\
\hline Atención & 19.18 & 23.41 & 174.5 & .264 \\
\hline Lectura & 19.63 & 23.04 & 183 & .361 \\
\hline Escritura & 18.84 & 23.7 & 168 & .199 \\
\hline
\end{tabular}

Nota. ${ }^{\mathrm{a}} \mathrm{n}=19 ;{ }^{\mathrm{b}} \mathrm{n}=23 .{ }^{*} p<.05$.

Psicomotricidad: problemas que involucran la diadococinesia y la desintegración sensorial -afectaciones en los movimientos a nivel motor fino o grueso-, y movimientos torpes y lentos.

Lenguaje expresivo: problemas articulatorios en el fonema $/ \mathrm{r} / \mathrm{y}$ errores de sustitución de este fonema por otro (p. ej., /n/; o /janinero/ por "jardinero"); morfologizaciones (p. ej., /plantador/ por "jardinero"); y mencionar otra palabra del mismo campo semántico (p. ej., /manzanas/ por "peras"). Dificultades que dan cuenta de inmadurez para la construcción adecuada de palabras, falta de estimulación en el nivel léxico y dificultades en la consolidación del mismo (sin embargo, no indican semiología neuropsicológica).

Fluidez verbal: problemas como la poca fluidez, o que la tarea de mencionar animales les pareció complicada, y que un minuto les parecía mucho tiempo (las dificultades eran más notorias conforme aumentaba la complejidad de la tarea). Los participantes mostraron dificultades para estructurar sintácticamente una sola oración coherente, congruente y con mayor número de palabras a partir de la presentación de dos palabras sin una relación clara (p. ej., televisión y tigre).

Visopercepción: problemas como trazo incoordinado y repaso de la unidad perceptual elaborada, dificultades relacionadas con la motricidad fina debido a fallos en la coordinación ojo-mano y la deficiencia en la prensión del lápiz (pinza); además de problemas al realizar la copia de figuras, donde destaca el tache y en donde el círculo, el cuadrado y el triangulo mostraban perseveraciones (dibujaban más de una vez el dibujo que se les solicitó).

Ritmo: problemas como que en el $69.6 \%$ de los casos los participantes no puedieron ejecutar correctamente los reactivos; sin embargo, no se puede concluir que los participantes no tengan ritmo, debido a que los puntajes que se obtuvieron en este rubro fueron resultado de factores asociados con la baja sensibilidad de la prueba para esta área.

\section{CUMANES (de 7 a 11 años)}

Para este grupo de edad se realizó un análisis de medidas de tendencia central y de dispersión de los centiles de cada subprueba del instrumento, donde se tomaron en cuenta los decatipos de la prueba del paciente estimada en percentiles -decatipos entendidos como la ejecución del participante: 10 como rendimiento muy alto y 1 como un rendimiento muy bajo-, obtenidos a partir de las puntuaciones tipificadas (Portellano et al., 2012). En la Tabla 6 se presentan los resultados de la ejecución de los participantes, donde se puede observar una mayor puntuación media en la subprueba de visopercepción $(M=6.18)$, y una menor puntuación media en escritura audioagnósica $(M=2.54)$.

Adicionalmente, en la Tabla 7 se presenta el porcentaje de los participantes que se ubican en el decatipo 4 o inferior -es decir, rendimiento bajo-, el porcentaje de niños en el decatipo 5 y $6-0$ rendimiento medio-y el decatipo 7 $-\mathrm{o}$ rendimiento alto-. Teniendo esto en cuenta, se observa que en la mayoría de las subpruebas el porcentaje más alto de niños se concentra en rendimiento bajo, y que las subpruebas con mayor concentración de participantes con bajo rendimiento son: ascritura audioagnósica (80.4\%), ritmo (69.6\%), comprensión de imágenes (65.7\%), fluidez semántica (63.7\%), leximetría-comprensión (67.7\%) y leximetría-velocidad (62.7\%). En contraste, el porcentaje más elevado con rendimiento alto se concentra en las 
Tabla 6.

Resultados de los decatipos obtenidos por los niños de 7 a 11 años en el CUMANES

\begin{tabular}{lcll}
\hline \multicolumn{1}{c}{ Subprueba } & $\mathrm{M}(\mathrm{DE})$ & $\mathrm{M}_{\mathrm{e}}$ & \multicolumn{1}{c}{$\mathrm{K}-\mathrm{S}$} \\
\hline Comprensión audioverbal & $5.48(2.46)$ & 5 & 1.199 \\
Comprensión de imágenes & $3.97(1.89)$ & 4 & $1.522^{*}$ \\
Fluidez fonológica & $4.44(1.99)$ & 4 & $1.363^{*}$ \\
Fluidez semántica & $3.86(1.95)$ & 4 & $1.433^{*}$ \\
Leximetría-comprensión & $3.70(1.99)$ & 4 & 1.18 \\
Leximetría-velocidad & $3.96(2.73)$ & 4 & 1.289 \\
Escritura audioagnósica & $2.54(1.98)$ & 1.5 & $2.839^{*}$ \\
Visopercepción & $6.18(2.03)$ & 6 & 1.214 \\
Función ejecutiva-tiempo & $4.44(2.23)$ & 4 & $1.38^{*}$ \\
Función ejecutiva-errores & $6.03(3.17)$ & 6 & $1.659^{*}$ \\
Memoria verbal & $4.10(1.85)$ & 4 & 1.174 \\
Memoria visual & $4.49(1.80)$ & 5 & 1.32 \\
Ritmo & $3.76(1.64)$ & 3 & $2.108^{*}$ \\
\hline
\end{tabular}

Nota. $M_{e}=$ mediana; $M=$ media; $D E=$ desviación estándar; $\mathrm{K}-\mathrm{S}=$ $\mathrm{z}$ de Kolmogorov-Smirnov. * significancia $<.05$ (no homogénea).

subpruebas: visopercepción (44.1\%), funciones ejecutivaserrores (43.1\%) y comprensión audio-verbal (37.3\%).

Por otro lado, en la Figura 1 se muestra en detalle la distribución de las dificultades tanto severas (decatipo 1) como moderadas (decatipo 2 y 3 ), donde se puede observar que la presencia de SNB está relacionada a procesos de lecto-escritura, ya que destacan tres subpruebas con un alto porcentaje de dificultades severas: (a) escritura audioagnósica (50\%), (b) leximetría-velocidad (25.5\%) y (c) leximetría-comprensión (19.6\%). Asimismo, se observan dificultades moderadas en las subescalas de ritmo (46.1\%), comprensión de imágenes (38.2 \%) y fluidez semántica (36.3\%); las dos últimas se relacionan con dificultades en procesos de lenguaje tanto expresivo como comprensivo.
Tabla 7.

Porcentaje de participantes con rendimiento bajo, medio y alto en las subpruebas del CUMANES (7 a 11 años)

\begin{tabular}{lccl}
\hline \multirow{2}{*}{\multicolumn{1}{c}{ Subprueba }} & \multicolumn{3}{c}{ Rendimiento } \\
\cline { 2 - 4 } & $\begin{array}{c}\text { Bajo } \\
(\%)\end{array}$ & $\begin{array}{c}\text { Medio } \\
(\%)\end{array}$ & $\begin{array}{c}\text { Alto } \\
(\%)\end{array}$ \\
\hline Comprensión audioverbal & 32.4 & 30.4 & 37.3 \\
Comprensión de imágenes & 65.7 & 24.5 & 9.8 \\
Fluidez fonológica & 53.1 & 29.4 & 16.7 \\
Fluidez semántica & 63.7 & 27.5 & 8.8 \\
Leximetría-comprensión & 67.7 & 27.5 & 9.8 \\
Leximetría-velocidad & 62.7 & 19.6 & 17.6 \\
Escritura audioagnósica & 80.4 & 15.7 & 3.9 \\
Visopercepción & 18.6 & 37.3 & 44.1 \\
Función ejecutiva-tiempo & 53.9 & 31.4 & 14.7 \\
Función ejecutiva-errores & 35.3 & 21.6 & 43.1 \\
Memoria verbal & 58.8 & 30.4 & 10.8 \\
Memoria visual & 48.0 & 39.2 & 12.7 \\
Ritmo & 69.6 & 21.6 & 8.8 \\
\hline
\end{tabular}

En lo que respecta a las diferencias según la variable sexo, se comparó cada subescala del CUMANES por medio de la prueba U de Mann Whitney, debido a que en la prueba de normalidad de Kolmogorov-Smirnov se encontró que la distribución de normalidad en las puntuaciones no era homogénea (Pérez-López, 2004). Cada una de las tareas presentó una distribución de los datos de manera no normal, en donde no se presentaron diferencias significativas en la ejecución de los niños. No obstante, cabe señalar que el grupo de niñas obtuvo puntuaciones medias mayores en las subpruebas de visopercepción $(M=7.00)$ y funciones ejecutivas-errores $(M=7.00)$ (véase Tabla 8$)$.

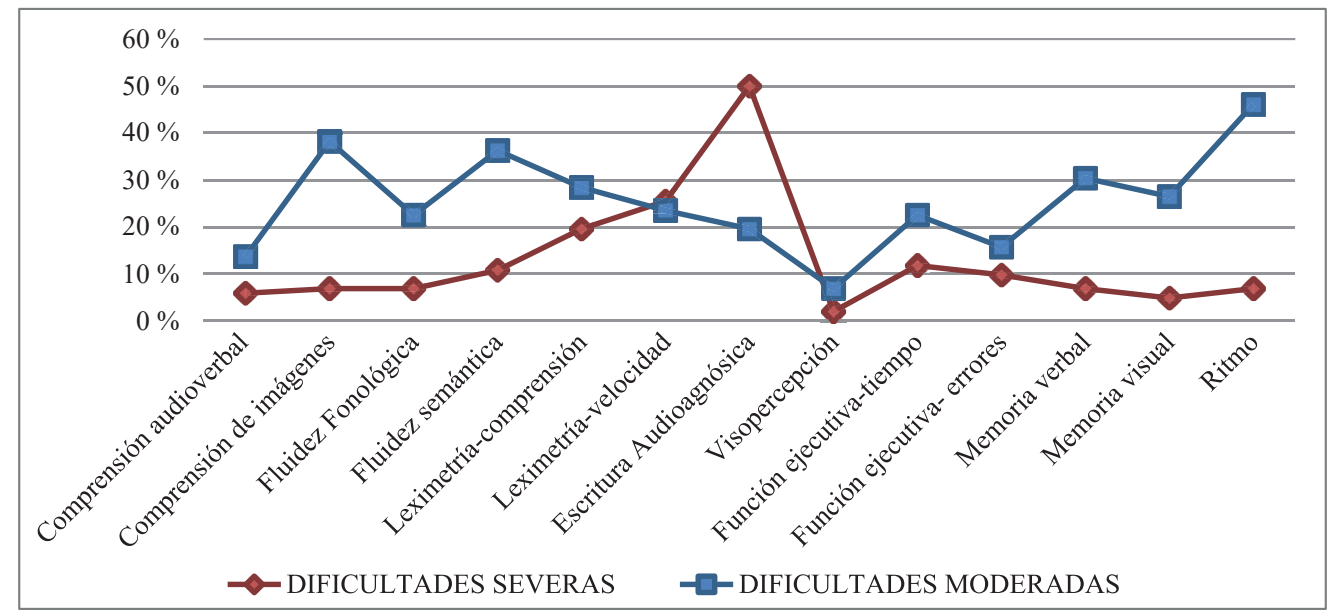

Figura 1. Porcentaje de niños con dificultades moderadas (decatipos 2 y 3 ) y dificultades severas (decatipo 1) del CUMANES. 
Tabla 8.

Rangos de la ejecución en el CUMANES según el sexo

\begin{tabular}{|c|c|c|c|c|}
\hline \multirow[t]{2}{*}{ Subprueba } & \multicolumn{2}{|c|}{$\begin{array}{c}\text { Sexo } \\
\text { (rango promedio) }\end{array}$} & \multirow{2}{*}{$\begin{array}{l}\text { U de Mann- } \\
\text { Whitney }\end{array}$} & \multirow[t]{2}{*}{$p$} \\
\hline & Niños $^{\mathrm{a}}$ & Niñas $^{\mathrm{b}}$ & & \\
\hline Comprensión audioverbal & 5.0 & 5.0 & 1158.5 & .379 \\
\hline Comprensión de imágenes & 4.0 & 4.0 & 1255.5 & .824 \\
\hline Fluidez fonológica & 4.0 & 4.5 & 1021 & .069 \\
\hline Fluidez semántica & 4.0 & 3.0 & 1031.5 & .081 \\
\hline Leximetría-comprensión & 4.0 & 4.0 & 1245 & .770 \\
\hline Leximetría-velocidad & 3.0 & 4.0 & 1081.5 & .160 \\
\hline Escritura audioagnósica & 1.0 & 2.0 & 1169.5 & .395 \\
\hline Visopercepción & 5.5 & 7.0 & 1067 & .133 \\
\hline Función ejecutiva-tiempo & 4.5 & 4.0 & 1270.5 & .905 \\
\hline Función ejecutiva-errores & 5.0 & 7.0 & 1114 & .238 \\
\hline Memoria verbal & 4.0 & 4.0 & 1253.5 & .814 \\
\hline Memoria visual & 5.0 & 5.0 & 1204.5 & .569 \\
\hline Ritmo & 3.0 & 3.0 & 1166 & .398 \\
\hline
\end{tabular}

Nota. ${ }^{\mathrm{a}} \mathrm{n}=56 ;{ }^{\mathrm{b}} \mathrm{n}=46$.

Por otra parte, con el fin de comparar los decatipos asociados a las diversas subpruebas del CUMANES entre los diferentes grupos de edad, se utilizó la prueba $\mathrm{H}$ de Kruskal-Wallis (véase Tabla 9), dado que en la prueba de normalidad Kolmogorov-Smirnov (subgrupos de edad) los datos se distribuyeron de manera no normal; a partir de esto, se encontraron diferencias significativas en las puntuaciones de cuatro subpruebas: leximetría-comprensión $\left(X^{2}=10.201, p<.05\right)$, función ejecutiva-errores $\left(X^{2}=16.412\right.$, $p<.05)$, memoria verbal $\left(X^{2}=11.283, p<.05\right)$ y ritmo $\left(X^{2}=14.168, p<.05\right)$.
Finalmente, el análisis cualitativo de los participantes que mostraron dificultades durante la ejecución de las actividades permitió caracterizar las principales problemáticas de este grupo de edad en las siguientes subpruebas:

Leximetría: los niños presentaron alteración de la comprensión lectora debido a dificultades de madurez fonológica, léxico pobre de acuerdo con la edad y morfologizaciones. La lectura en voz alta se caracterizó por fallos asociados en signos de puntuación y en la velocidad de lectura.

Visopercepción: en general, la ejecución de los participantes presentó un desarrollo adecuado para la edad.

Tabla 9.

Promedio de ejecución en las subpruebas del CUMANES según la edad

\begin{tabular}{|c|c|c|c|c|c|c|c|}
\hline \multirow{2}{*}{ Subprueba } & \multicolumn{5}{|c|}{ Edad } & \multirow{2}{*}{$X^{2}(4)$} & \multirow{2}{*}{$p$} \\
\hline & 7 años ${ }^{\mathrm{a}}$ & 8 años ${ }^{b}$ & 9 años ${ }^{c}$ & 10 años ${ }^{\mathrm{d}}$ & 11 años ${ }^{\mathrm{e}}$ & & \\
\hline Comprensión audioverbal & 4.0 & 5.0 & 6.5 & 6.0 & 6.5 & 8.384 & .078 \\
\hline Comprensión de imágenes & 4.0 & 4.0 & 3.0 & 4.0 & 4.0 & 2.403 & .662 \\
\hline Fluidez fonológica & 4.0 & 4.0 & 4.0 & 5.0 & 4.5 & 2.082 & .721 \\
\hline Fluidez semántica & 4.0 & 3.0 & 4.0 & 3.0 & 4.0 & 1.198 & .878 \\
\hline Leximetría-comprensión & 2.5 & 4.0 & 4.0 & 3.0 & 5.5 & 10.201 & $.037 *$ \\
\hline Leximetría-velocidad & 2.5 & 4.0 & 4.0 & 4.0 & 3.0 & 2.301 & .681 \\
\hline Escritura audioagnósica & 1.0 & 2.0 & 4.0 & 2.0 & 1.0 & 6.461 & .167 \\
\hline Visopercepción & 5.5 & 6.0 & 6.0 & 7.0 & 5.5 & 9.118 & .058 \\
\hline Función ejecutiva-tiempo & 5.0 & 5.0 & 4.0 & 4.0 & 4.0 & 1.331 & .856 \\
\hline Función ejecutiva-errores & 8.0 & 9.0 & 4.0 & 5.0 & 4.5 & 16.412 & $.003 *$ \\
\hline Memoria verbal & 5.0 & 4.0 & 4.0 & 3.0 & 2.0 & 11.283 & $.024 *$ \\
\hline Memoria visual & 4.0 & 5.0 & 4.0 & 5.0 & 5.0 & 1.725 & .786 \\
\hline Ritmo & 4.0 & 4.0 & 3.0 & 2.0 & 2.5 & 14.168 & $.007 *$ \\
\hline
\end{tabular}

Nota. ${ }^{\mathrm{a}} \mathrm{n}=26 ;{ }^{\mathrm{b}} \mathrm{n}=35 ;{ }^{\mathrm{c}} \mathrm{n}=14 ;{ }^{\mathrm{d}} \mathrm{n}=15 ;{ }^{\mathrm{e}} \mathrm{n}=12 .{ }^{*} \mathrm{p}<.05$. 
Sin embargo, a los 11 años se observa una disminución en la ejecución, probablemente porque las estrategias de elaboración de estímulos a esta edad cambian o se están adaptando a la exigencia de los procesos cognitivos.

Funciones ejecutivas: independientemente de la edad, los niños realizaron un rastreo desorganizado, reflejado en la cantidad de errores cometidos, ya sea por falta de organización o planeación. Los niños de 9, 10 y 11 años tendieron a equivocarse de manera más frecuente, y es en estas edades donde pruebas como funciones ejecutivaserrores, memoria verbal y ritmo presentan un descenso en su ejecución.

\section{Discusión}

El propósito de la presente investigación fue evaluar y comparar la presencia de SNB con los procesos cognitivos superiores de niños escolares mexicanos de un estrato socioeconómico medio-bajo sin presencia de antecedentes neurológicos o psiquiátricos. Los hallazgos muestran la presencia de signos psicomotores, como el trastorno en la integración sensorial, la disdiadococinesia y el trastorno de coordinación; así como signos perceptivos, como el trastorno de orientación espacial y la dificultad en el reconocimiento de derecha-izquierda; signos relacionados con procesos como la atención, la memoria, el razonamiento, el lenguaje (oral, comprensivo y escrito), y dificultades de aprendizaje.

Algunos de estos SNB debieron desaparecer en etapas tempranas del desarrollo para dar pauta a conductas más complejas que se exigen dentro del medio escolar y social. Sin embargo, tras la exploración neuropsicológica, los niños de 6 años evaluados en el presente estudio mostraron dificultades en áreas específicas, como en la psicomotricidad; sin embargo, en esta edad se espera que mejoren tanto su capacidad motora gruesa -y puedan dar saltos breves, lanzar una pelota con cierto peso y tamaño-como su nivel motor fino -y puedan tomar un lápiz para dibujar e, incluso, abotonarse una camisa- (Feldamn, 2007).

En concordancia con estos resultados, Ozkan, Kara, Mahmoud y Congologu (2018) observaron que su muestra de participantes, clasificados con funcionamiento intelectual límite, a diferencia de pacientes con diagnóstico de desorden específico del aprendizaje, presentaron mayor número de SNB, principalmente en la integración sensorial, la coordinación motora y la valoración total del instrumento utilizado por los investigadores.

De igual forma, en el presente trabajo se encontró una disritmia-definida como un tiempo o ritmo del movimiento incorrectos- que, de acuerdo con Pitizianti et al. (2017), junto con la alteración del ritmo de las respuestas motoras y la mala coordinación motriz, parecen ser las anormalidades motoras más prominentes en niños con alteraciones en el desarrollo del sistema nervioso y el TDAH. Esto coincide con lo señalado por Ávila (2012) y Parra-Pulido, RodríguezBarreto y Chinome-Torres (2016), cuyos resultados indican la presencia de SNB como posibles indicadores de inmadurez del Sistema Nervioso Central que repercuten en el desarrollo intelectual, conductual y emocional de los niños.

Por otra parte, en los niños de 6 años de este trabajo también se encontró una ejecución deficiente de la prueba visoperceptiva, aun cuando se espera que en esta edad el desarrollo de áreas corticales occipitales-parietales tengan una mayor integración de los estímulos visuales. Como señalan Capellini, Giaconi y Germano (2017), la actividad visomotriz se define como una integración fluida entre la capacidad visoperceptual y las habilidades motoras, lo que requiere la capacidad de traducir la percepción visual a la función motora, o la capacidad de coordinar la mano y el ojo, lo cual tiene un papel importante en el futuro desarrollo académico del niño.

En cuanto a los procesos cognitivos, se encontraron alteraciones a nivel del lenguaje expresivo-articulatorio y comprensivo, a pesar de que, de acuerdo con Narbona y Chevrie-Muller (2003), a la edad de 6 años se consolidan todos los fonemas -exceptuando el fonema/r/- en lo concerniente a la comprensión, el infante puede entender más del doble de vocabulario del que expresa, además de que aumenta la complejidad en la comprensión de estructuras sintácticas que utiliza. Igualmente, se encontró alteración en la fluidez verbal, a pesar de que el niño a esta edad debe ser capaz de generar entre 10 y 16 palabras dentro de un campo semántico (García et al., 2012); además de un detrimento en la atención, a pesar de que los niños de esta edad pueden concentrarse durante más tiempo y enfocarse en la información necesaria y filtrar la innecesaria (Anderson, Jacobs \& Anderson, 2010).

Los hallazgos encontrados en este aspecto concuerdan con los datos publicados por Mateos y López (2011) con respecto a que la presencia de trastornos psicomotores, sensoriales y cognitivos en niños en etapa escolar son un factor de riesgo para la presencia de trastornos de lenguaje expresivo (hablado y escrito), trastornos en la fluidez semántica, problemas en lectura -escaso ritmo y velocidad lectora-, y pobre o nula comprensión del texto leído; dificultades visoperceptivas que pueden influir en un desarrollo anómalo de la escritura y el cálculo.

Por otro lado, al analizar el desempeño en las subpruebas de CUMANIN y la variable de sexo del participante se encontraron diferencias significativas en el lenguaje expresivo, con un mejor desempeño en las niñas; resultados que contrastan con los obtenidos por Ávila (2012), 
Parra-Pulido et al. (2016), Portellano et al. (2009) y Urzúa, Ramos, Alday y Alquinta (2010), en los cuales se indica que la presencia de SNB no se asocia con la variable sexo durante esta etapa del desarrollo.

Adicionalmente, los resultados en la ejecución de niños de 7 a 11 años en el CUMANES ponen de manifiesto la presencia de SNB relacionados con la escritura audioagnósica, la leximetría-velocidad y la leximetría-comprensión de lenguaje. Los resultados observados en las comparaciones por edad indicaron diferencias significativas en áreas del lenguaje, funciones ejecutivas, memoria verbal y ritmo, lo cual puede deberse a las estrategias cognitivas desarrolladas que cambian en función de algunos puntos críticos en el desarrollo.

Al respecto, Battro, Fischer y Lena (2016) destacan la importancia de estadios críticos durante los cuales se activan más los procesos de maduración cerebral y se desarrollan con mayor intensidad las conexiones nerviosas; periodos que se presentan alrededor de los 4, 8 y 12 años de edad. Aunado a ello, las exigencias del medio ambiente en el que se desarrollan los niños y la demanda cognitiva escolar juegan un papel importante en la adaptación y el desarrollo de nuevas estrategias. Adicionalmente, Chadwik (1988), por ejemplo, señala que estos cambios de estrategia cognitiva pueden darse en dos niveles: el nivel de procesamiento, que incluye las estrategias que la persona usa normalmente en forma automática para mejorar sus posibilidades de ingresar y almacenar información, impresiones, datos específicos, ideas generales, etc.; y el nivel de ejecución, que incluye todo lo que la persona hace para recuperar o resolver problemas y generar respuestas creativas. La diferencia entre los dos niveles puede ser vista como estrategias de entrada y de salida.

En cuanto a la escritura audioagnósica en la prueba de CUMANES, se requiere que el niño realice un escrito dictado por el evaluador, tarea cognitiva que permite saber si conserva un reconocimiento auditivo verbal adecuado y si aporta información relevante en el desarrollo temprano del funcionamiento de la memoria de trabajo; aspecto que juega un papel importante en la capacidad para procesar y realizar tareas cognitivas complejas de tipo auditivo verbal, como leer y escribir (Tindle \& Longstaff, 2015).

Con respecto a la leximetría-comprensión y leximetríavelocidad, los niños del presente estudio presentaron un déficit en la ejecución de esta tarea, la cual, cognitivamente hablando, es una función relevante asociada a la habilidad lectora y, por tanto, para el aprendizaje, debido a que en este se requiere que la atención de los lectores se centre en la comprensión mientras que la mecánica del reconocimiento de palabras opera con velocidad y de manera automática (Abu-Leil, Share \& Ibrahim, 2014).
Ahora bien, con respecto a la asociación de factores sociodemográficos, en el presente estudio la muestra resultó ser homogénea, lo que impidió realizar el contraste a nivel estadístico. Sin embargo, Urzúa et al. (2010) señalan que el nivel socioeconómico determina las diferencias en el desempeño de las subpruebas del CUMANIN; y la UNESCO (2015) manifiesta que estudiantes con mejor rendimiento académico provienen de un estrato socioeconómico alto. De la misma manera, se ha encontrado que un bajo nivel educativo de los padres se relaciona con bajo rendimiento escolar de sus descendientes (Matute, Sanz, Gumá, Roselli \& Ardila, 2009), al igual que otros factores, como la pobreza, hábitos de vida, modelos de interacción familiar, la comunicación lingüística al interior del hogar y las expectativas de educación hacia los hijos. La limitación del presente estudio se relaciona con la procedencia de los participantes y su nivel socioeconómico. Una muestra derivada de la población mexicana de tipo heterogénea (áreas urbanas y rurales) hubiera enriquecido los resultados obtenidos.

La justificación de este estudio se relaciona con el escaso número de investigaciones en las que se hace uso de la batería CUMANES, y con la poca observación y evaluación de los SNB (Becerra-Garrido, 2016), por lo que sería relevante un análisis a profundidad en la investigación de este tema, sobre todo estimando la validez ecológica que implican los resultados de la investigación, dado que se debe evaluar la conducta del individuo en un contexto con múltiples niveles de influencia, donde destaca el intrapersonal (biológico y psicológico), el interpersonal (social y cultural), y las organizaciones, las comunidades y el ambiente físico y político de las personas (Salvador et al., 2016).

Por último, cabe mencionar que el fracaso escolar puede ocurrir por múltiples factores, entre los cuales destacan el nivel socioeconómico y la presencia de $\mathrm{SNB}$, por lo que la identificación y diagnóstico en etapas tempranas del desarrollo de los SNB permitirá anticipar el rendimiento académico del niño al desarrollar líneas de intervención secundaria o estimulación temprana que impidan el futuro detrimento de la actividad cognitiva durante el desarrollo y se pueda, de este modo, mejorar su calidad de vida en el ámbito escolar.

\section{Referencias}

Abdel-Aziz, A., El Sheikh, M., Mohsen, M., Khalil, A., \& Hassan, A. (2016). Neurological soft signs in a sample of Egyptian ADHD children and their clinical correlates. Middle East Current Psychiatry, 23(2), 51-55. doi: 10.1097/01.XME.0000481458.63018.89 
Abu-Leil, A. K., Share, D., \& Ibrahim, R. (2014). How does speed and accuracy in reading relate to reading comprehension in Arabic? Psicológica, 35, 251-276. Recuperado de https://www.uv.es/revispsi/articulos2.14/5ABULEIL.pdf

Anderson, V., Jacobs, R., \& Anderson, P. J. (Eds.). (2010). Executive Functions and the Frontal Lobes: A Lifespan Perspective. New York: Psychology Press.

Ávila, A. (2012). Adaptación del cuestionario de Madurez Neuropsicológica Infantil CUMANIN de Portellano. Revista Iberoamericana de Psicología: Ciencia y Tecnología, 5(1), 91-100. Recuperado de http://ibero-revistas.metabiblioteca. org/index.php/ripsicologia/article/view/239/207

Battro, A. M., Fischer, K. W., \& Lena, P. J. (2016). El cerebro educado: Bases de la Neuroeducación. Argentina: Gedissa.

Becerra-Garrido, P. (2016). La influencia de la ansiedad en la función ejecutiva en niños: Estudio piloto con alumnos de primaria en un centro de Badajoz (tesis de maestría). Universidad de Extremadura, España. Recuperado de http://dehesa.unex.es/bitstream/handle/10662/4364/ TFMUEX_2016_Becerra_Garrido.pdf? sequence $=1$

Bombin, I., Arango, C., \& Buchanan, R. W. (2005). Significance and Meaning of Neurological Signs in Schizophrenia: Two Decades Later. Schizophrenia Bulletin, 31(4), 962-977. doi: https://doi.org/10.1093/schbul/sbi028

Capellini, S.A., Giaconi, C. \& Germano, G. (2017). Relation between visual motor integration and handwriting in students of elementary school. Psychology, 8, 258-270. doi: 10.4236/psych.2017.82015

Cardo, E., Casanovas, S., Banda, G. D., \& Servera, M. (2008). Signos neurológicos blandos: ¿tienen alguna utilidad en la evaluación y diagnóstico del trastorno por déficit de atención/hiperactividad? Revista de Neurología, 46 (1), 51-54. doi: https://doi.org/10.33588/rn.46S01.2008005

Chadwick, C. B. (1988). Estrategias Cognoscitivas y Afectivas de Aprendizaje. Revista Latinoamericana de Psicología, 20(2), 163-205. Recuperado de http://www.redalyc.org/ pdf/805/80520203.pdf

Chan, R. C. K., Xie, W., Geng, F., Wang, Y., Lui, S. S. Y., ... Rosenthal, R. (2016). Clinical Utility and Lifespan Profiling of Neurological Soft Signs in Schizophrenia Spectrum Disorders. Schizophrenia Bulletin, 42(3), 560-570. doi: 10.1093/schbul/sbv196

Chetail-Vijay, D., \& Shubhangi, P. (2016). Soft Neurological Signs and cognitive function in Obsessive-compulsive disorder patients. Indian Journal of Psychological Medicine, 38(4), 291-295. doi: 10.4103/0253-7176.185957

Chrobak, A. A., Siwek, G. P., Siuda-Krzywicka, K., Arciszewska, A., Starowicz-Filip, A., Siwek, M., \& Dudek, D. (2016). Neurological and cerebellar soft signs do not discriminate schizophrenia from bipolar disorder patients. Progress in Neuro-Psychopharmacology and Biological Psychiatry, 64, 96-101. doi: 10.1016/j.pnpbp.2015.07.009

Coolican, H. (2014). Research methods and statistics in psychology (6. ${ }^{\mathrm{a}}$ ed.). Psychology Press.
Dhuri, C. V., \& Parkar, S. R. (2016). Soft Neurological Signs and Cognitive Function in Obsessive Compulsive Disorder Patients. Indian Journal of Pharmacology, 38(4), 291-295. doi: 10.4103/0253-7176.185957

Emsley, R., Chiliza, B., Asmal, L., Kilian, S., Riaan-Olivier, M., ... Dazzan, P. (2017). Neurological soft signs in firstepisode schizophrenia: State- and trait_related relationships to psychopathology, cognition and antipsychotic medication effects. Schizophrenia Research, 188, 144-150. doi: 10.1016/j.schres.2017.01.034

Faruk-Demirel, O., Demirel, A., Tayibb-Kadak, Emül, M., \& Duran, A. (2016). Neurological Soft Signs in antisocial men and relation with psychopathy. Psychiatry Research, 248252. doi: 10.1016/j.psychres.2016.04.094

Feldamn, R. (2007). Desarrollo psicológico, a través de la vida. Ciudad de México: Pearson Prentice Hall.

García, E., Rodríguez, C., Martín, R., Jímenez, J., Hernández, S., \& Díaz, A. (2012). Test de Fluidez Verbal: datos normativos y desarrollo evolutivo en el alumnado de primaria. European Journal of Education and Psychology, 5(1), 53-64. Recuperado de http://www.redalyc.org/articulo. oa?id $=129324775005$

Hermida, M., Segretin, M., Lipina, S., Benarós, S., \& Colombo, J. (2010). Abordajes neurocognitivos en el estudio de la pobreza infantil: consideraciones conceptuales y metodológicas. International Journal of Psychology and Psychological Therapy, 10(2), 205-225. Recuperado de http://www.ijpsy. $\mathrm{com} /$ volumen10/num2/258/abordajes-neurocognitivos-enel-estudio-ES.pdf

Hirjak, D., Thomann, P., Wolf, R., Kubera, K., Goch, C., Hering, J., \& Maier-Hein, K. (2017). White matter microestructure variations contribute to neurological soft signs in healthy adults. Human Brain Mapping, 38(7), 1-14. doi: 10.1002/ hbm. 23609

Instituto Nacional de Estadística y Geografía (INEGI). (2015). Índice de Población. Recuperado de http://www.beta.inegi. org.mx/temas/estructura/

Jingbo-Gong, Jingtao-Xie, G., Yajie-Zhang, \& Su-Hong-Wang. (2015). Neurological soft signs in children with attention deficit hyperactivity disorder: Theirrelationship to executive function and parental neurological soft signs. Psychiatry Research, 77-82. doi: https://doi.org/10.1016/j.psy chres.2015.04.017

Kerlinger, F. (2002). Investigación del comportamiento. Métodos de investigación en ciencas sociales. Ciudad de México: McGraw-Hill

Lipina S. (2016) Pobre cerebro: los efectos de la pobreza sobre el desarrollo cognitivo y emocional, y lo que la neurociencia puede hacer para prevenirlos. Buenos Aires: Siglo XXI.

Malhotra, S., Borade, P., Sharma, P., Satija, Y., \& Gunjan. (2017). A qualititative study of neurological soft signs in obsessive compulsive disorder and effect of comorbid psychotic spectrum disorders and familiality on its expression 
in Indian population. Asian J Psychiatr, 25, 6-12. doi: 10.1016/j.ajp.2016.06.020

Manaut-Gil, E., Vaquero-Casares, E., Quintero-Gallego, E., Pérez-Santamaría, J., \& Gómez-González, C. M. (2004). Relación entre el déficit neurológico y el cociente de inteligencia en niños y adolescentes. Revista de Neurología, 38(1), 20-27. Recuperado de https://pdfs.semanticscholar. org/f9b1/b0ca1a26915a8ff441189167508d8a4b0efd.pdf

Mateos, R. (2009). Dificultades de aprendizaje. Psicología Educativa, 15(1), 13-19. Recuperado de http://www.ujaen. es/revista/rei/linked/documentos/documentos/12-7.pdf

Mateos, R., \& López, C. (2011). Dificultades de aprendizaje: Problemas del diagnóstico tardío y/o del infradiagnóstico. Revista Educación Inclusiva, 4(1), 103-111. Recuperado de http://www.ujaen.es/revista/rei/linked/documentos/docu mentos/12-7.pdf

Matute, E., Sanz, A., Gumá, E., Roselli, M., \& Ardila, A. (2009). Influencia del nivel educativo de los padres, el tipo de escuela y el sexo en el desarrollo de la atención y la memoria. Revista Latinoamericana de Psicología, 42(2), 257-276. Recuperado de http://www.redalyc.org/ pdf/805/80511496006.pdf

Mazzoni, C., Stelzer, F., Cervigni, M., \& Martino, P. (2014). Impacto de la pobreza en el desarrollo cognitivo. Un análisis teórico de dos factores mediadores. Libert, 20(1), 93-100. Recuperado de http://www.scielo.org.pe/scielo. php?script=sci_arttext\&pid=S1729-48272014000100008

Mithun, D., Kamal, N., Aparajeeta, B., \& Subrata, N. (2016). A clinical study of neurological soft signs in patients with schizophrenia. Journal of Neurosciencies in Rural Practice, 7(3), 393-399. doi: 10.4103/0976-3147.181481

Narbona, J., \& Chevrie-Muller, C. (2003). El lenguaje del niño, desarrollo normal, evaluación y trastornos (2. ${ }^{a}$ ed.). Barcelona: Masson.

Ojagbemi, A. (2017). Neurological Soft Signs. En V. ZeiglerHill \& T. Shackelford, Encyclopedia of Personality and Individual Diferences. EE. UU.: Springer Cham.

Organización de las Naciones Unidas para la Educación, la Ciencia y la Cultura (UNESCO). (2015). Informe de seguimiento de la educación para todos en el mundo. Recuperado de http://unesdoc.unesco.org/ images/0023/002324/232435s.pdf

Ozkan, S., Kara, K., Mahmoud, A., \& Congologu, M. (2018). Investigation of distinctive characteristics of children with specific learning disorder and borderline intellectual functioning. Archives of clinical psychiatry 45(1), 1-6. doi: 10.1590/0101-60830000000145

Papiol, S., Fatjo-Vilas, M., \& Shulze, T. (2016). Neurologic soft signs in patients with schizophrenia: current knowledge and future perspectives in the post genomics era. Translational Developmental Psychiatry, 4(1), 1-10. doi: http://dx.doi. org/10.3402/tdp.v4.30071
Parra-Pulido, H., Rodríguez-Barreto, L., \& Chinome-Torres, J. (2016). Evaluación de la madurez neurospicológica infantil en preescolares. Revista Universidad y Salud, 18(1), 126-137. Recuperado de http://www.scielo.org.co/pdf/reus/ v18n1/v18n1a13.pdf

Patankar, V., Sangle, J., Henal, R., Shah, D., \& Kamath, R. (2012). Neurological soft signs in children with attetion deficit hiperactivity disorder. Indian Journal of psychiatry, 54(2), 159-165. doi: 10.4103/0019-5545.99540

Peng, Z., Xu, T., Miao, G., He, Q., Zhao, Q., Dazzan, P., \& Chan, R. (2012). Neurological soft sings in obsessive-compulsive disorder: The effect of co-morbid psychosis and evidence for familiality. Progress in Neuro-Psychopharmacology and Biological Psychiatry, 39(1), 200-205. doi: 10.1016/j. pnpbp.2012.06.015

Pérez-López, C. (2004). Técnicas de Análisis Multivariante de Datos. Madrid, España: Pearson Educación, S. A.

Pitizianti, M., Grelloni, C., Casarelli, L., D'Agati, E., Spiridigliozzi, S., Curatolo, P., \& Pasini, A. (2017). Neurological soft signs but not theory of mind and emotion recognition deficit distinguished children with ADHD from healthy control. Pschiatry Research, 256, 1-20. doi: 10.1016/j.psychres.2017.06.029

Poblano, A., Borja, S., Elías, Y., García-Pedroza, F., \& Arias, M. D. L. (2002). Characteristics of specific reading disability in children from a neuropsychological clinic in Mexico City. Salud Publica de México, 44(4), 323-327. doi: 10.1590/ S0036-36342002000400005

Portellano, J., Mateos, R. M., \& Martínez, A. (2009). Cuestionario de Madurez Neuropsicológica Infantil: CUMANIN (4. ${ }^{\mathrm{a}}$ ed.). Madrid: TEA Ediciones.

Portellano, J., Mateos, R., \& Martínez, R. (2012). Cuestionario de Madurez Neuropsicológica Escolar: CUMANES. Madrid: TEA Ediciones.

Salvador, J., Armengol, C., García, A., Aguillón, C., Licerio, E. A., Sánchez, E., \& Cuéllar, C. (2016). Modelo de validez ecológica en la práctica neuropsicológica: problemas neurológicos y/o psiquiátricos, y de aprendizaje hacia la rehabilitación neuropsicológica integral. En M. A. Padilla, S. Galán, E. Camacho \& A. Zarate (eds.), Investigación en psicología básica y aplicada: avances y perspectivas (pp. 91-97). México: Universidad de Guadalajara.

Salvador, J., \& Galindo, G. (1996). Cuestionario de antecedentes neurológicos y psiquiátricos. En Diseño de un nuevo procedimiento para calificar la prueba de la figura compleja de Rey: confiabilidad inter-evaluadores. Salud Mental, 19(2), 1-6. Recuperado de http://revistasaludmental.mx/in dex.php/salud_mental/article/view/585/585

Somale, A., Kondekar, S., Rathi, S., \& Iyer, N. (2016). Neurodevelopmental comorbidity profile in specific learning disorders. International Journal of Contemporary Pediatrics , 355-361. doi: http://dx.doi.org/10.18203/23493291.ijcp20160836 
Tindle, R., \& Longstaff, M. (2015). Writing, Reading, and listening differentially overload working memory performance across the seral position curve. Advances in Cognitive Psychology, 11(4), 147-155. doi: 10.5709/acp-0179-6

Torres-González, C., Salvador-Cruz, J., Flores, J., \& RicardoGarcell, J. (2016). Inteligencia general en niños nacidos prematuramente. Cuadernos de Neuropsicología. Panamerican Journal of Neuropsychology, 10(2), 142-164. doi: $10.7714 / \mathrm{CNPS} / 10.2 .208$
UNICEF México. (s. f.). UNICEF México:Presentan UNICEF y CONEVAL análisis sobre pobreza y derechos sociales de niñas, niños y adolescentes en México. Recuperado de https:// www.unicef.org/mexico/spanish/noticias_33097.html

Urzúa, A., Ramos, M.,Alday, C., \& Alquinta, A. (2010). Madurez neuropsicológica en preescolares: Propiedades psicométricas del test CUMANIN. Terapia Psicologica, 28(1), 13-25. doi: https://doi.org/10.4067/S0718-48082010000100002 